\title{
Asociación entre conducta suicida y sintomas de anorexia y bulimia nerviosa en escolares de Boyacá, Colombia
}

\author{
Lizeth Cristina Martínez Baquero ${ }^{1 *}$, Mildred Alexandra Vianchá Pinzón ${ }^{1}$, Mónica Patricia Pérez Prada \\ y Bertha Lucia Avendaño Prieto ${ }^{2}$ \\ ${ }^{1}$ Universidad de Boyacá, ${ }^{2}$ Universidad Católica de Colombia
}

Recibido, abril 13/2016

Concepto de evaluación, febrero 28/2017

Aceptado, mayo 5/2017
Referencia: Martínez Baquero, L.C., Vianchá Pinzón, M.A., Pérez Prada, M.P. \& Avendaño Prieto, B.L. (2017). Asociación entre conducta suicida y síntomas de anorexia y bulimia nerviosa en escolares de Boyacá, Colombia. Acta Colombiana de Psicología. 20(2), 178-188. doi: http:// www.dx.doi.org/10.14718/ACP.2017.20.2.9

Resumen

La presente investigación tuvo como objetivo establecer la asociación existente entre síntomas de trastornos alimentarios y la ideación e intento suicida en adolescentes de grado octavo a undécimo de instituciones educativas de tres ciudades del departamento de Boyacá, Colombia. Se realizó una estrategia descriptiva con un diseño selectivo probabilístico transversal. La muestra estuvo conformada por 1292 participantes seleccionados mediante muestreo probabilístico estratificado. Con el fin de indagar los factores protectores y de riesgo para la ideación suicida, la presencia de acto suicida y el tamizaje de trastornos de la conducta alimentaria, se aplicó el cuestionario PANSI (Osman, Gutiérrez, Kopper, Barrios \& Chiros, 1998), la encuesta sociodemográfica elaborada por las autoras y el SCOFF (Morgan, Reid \& Lacey, 1999), respectivamente. Los resultados muestran que del total de participantes, el $17.8 \%$ (230) piensan en el suicidio como alternativa y que el $42 \%$ de quienes registran sintomatología relacionada con trastornos alimentarios presentan ideación suicida negativa (riesgo). En conclusión, se identificó una correlación baja, pero significativa, entre los trastornos alimentarios y el PANSI negativo ( $\mathrm{rs}=.19 \mathrm{y} \mathrm{p}=.00$ ), lo que sugiere que a mayor presencia de señales de alarma de trastornos alimentarios, más factores de riesgo de ideación suicida.

Palabras clave: Ideación suicida, intento suicida, trastornos alimentarios, adolescentes.

\section{Association between symptoms of anorexia and bulimia nervosa and suicidal behavior in school children of Boyacá, Colombia}

\begin{abstract}
This research aimed to establish the relationship between eating disorders and suicidal ideation and attempt, in adolescents from eighth to eleventh grade of educational institutions in three cities of Boyacá (Colombia). The study conducted was empirical and used quantitative methodology. A descriptive strategy was implemented using a cross-sectional design. The sample consisted of 1292 participants, selected by stratified probability sampling. The instruments applied were firstly, the Positive and Negative Suicidal Ideation PANSI Inventory (Osman, Gutiérrez, Kopper, Barrios and Chiros, 1998) to inquire about the protective and risk factors for suicidal ideation and the presence of suicidal act. Secondly, the Sick Control on Fat Food -SCOFF Questionnaire (Morgan, Reid \& Lacey, 1999) to carry out a screening for eating disorders. Thirdly, a sociodemographic survey designed by the authors, to characterize the sample of participants. Results show that out of the total number of young people who participated in the study, $230(17.8 \%)$ consider suicide as an alternative. The research identified low but significant correlation between eating disorders and negative PANSI ( $\mathrm{rs}=.19$ and $\mathrm{p}=0.00$ ), suggesting that the higher the presence of alarm signals of eating disorders, the higher the risk factors for suicidal ideation.

Key words: Eating disorders, suicidal ideation, suicide attempt, adolescents.
\end{abstract}

* Carrera 2 A Este, No. 64-169, (+57) 7450000, lcmartinez@uniboyaca.edu.co 


\title{
Associação entre comportamento suicida e sintomas de anorexia e bulimia nervosa em estudantes escolares de Boyacá, Colômbia
}

\begin{abstract}
Resumo
Esta pesquisa teve como objetivo estabelecer a associação existente entre sintomas de transtornos alimentares e ideação suicida e sua tentativa em adolescentes de instituições educativas de três cidades do estado de Boyacá, Colômbia. Realizou-se uma estratégia descritiva com um desenho seletivo probabilístico de corte transversal. A amostra foi conformada por 1292 participantes selecionados mediante amostragem probabilística estratificada. A fim de indagar os fatores protetores e de risco para a ideação suicida, a presença de ato suicida e o triagem de transtornos do comportamento alimentar, aplicaram-se o questionário PANSI (Osman, Gutiérrez, Kopper, Barrios \& Chiros, 1998), a enquete sociodemográfica elaborada pelas autoras e o SCOFF (Morgan, Reid \& Lacey, 1999), respectivamente. Os resultados mostraram que, do total de participantes, $17.8 \%$ (230) pensam no suicídio como alternativa e que $42 \%$ dos que registram sintomatologia relacionada com transtornos alimentares apresentam ideação suicida negativa (risco). Em conclusão, identificou-se uma correlação baixa, mas significativa, entre os transtornos alimentares e o PANSI negativo ( $\mathrm{rs}=.19$ e $\mathrm{p}=.00)$, o que sugere que, quanto maior presença de sinais de alarme de transtornos alimentares, mais fatores de risco de ideação suicida há.

Palavras-chave: Adolescentes, ideação suicida, tentativa suicida, transtornos alimentares.
\end{abstract}

\section{INTRODUCCIÓN}

La adolescencia puede entenderse como un periodo de transición que hace posible el paso de la infancia a la edad adulta; comienza con la pubertad (10-12 años) y finaliza entre los 19 y 20 años de edad (Galdó, 2008; Quintero \& Piñeros, 2010); se trata de una etapa en la que ocurren cambios rápidos e importantes en áreas biológicas, psicológicas y sociales, pues se tienen las tareas de la búsqueda y consolidación de la identidad, además del logro de la autonomía; y su culminación implica alcanzar la madurez y la independencia (Gaete, 2015).

En Colombia, según el informe "Forensis, datos para la vida, 2015" del Instituto Nacional de Medicina Legal y Ciencias Forenses (INML, 2015), se ha identificado que el departamento de Boyacá cuenta con dos de los municipios con mayor tasa de suicidio por cada 100000 habitantes; y según dicho informe, pero en años anteriores (INML, 2012), este departamento es el segundo con mayor tasa de suicidios en el país, en donde la población adolescente resulta ser la más vulnerable.

El suicidio, definido como el acto con resultado fatal, anticipado y llevado a cabo por una persona con conocimiento y propósito de obtener un cambio deseado (WHO, 2012), tiene una estrecha relación con evitar la humillación y las situaciones vergonzosas, según los relatos de jóvenes con que han intentado suicidarse (Cortés, 2014). Y de acuerdo con Córdova, Cubillas y Román (2011), la conducta suicida es un proceso que inicia con la idea de suicidarse, pasa por el intento de suicidio, y, en algunos casos, concluye con la muerte auto infligida, es decir, el suicidio consumado.
Específicamente, las ideas suicidas pueden abarcar desde pensamientos de falta de aprecio por la vida hasta la planificación de un acto letal. Estas ideas pueden pasar por deseos más o menos intensos de muerte en los que intervienen procesos cognitivos y afectivos que varían desde sentimientos sobre la falta del sentido de vivir y la elaboración de planes para quitarse la vida, hasta preocupaciones sistemáticas y delirantes (Ballesteros et al., 2010; Casullo, Bonaldi \& Fernández, 2000; Portilla, 2006).

Esta ideación se presenta "cuando un sujeto persistentemente piensa, planea, o desea cometer suicidio, durante por lo menos dos semanas, haciendo algún plan e identificando los medios necesarios para conseguirlo" (Mingote, Jiménez, Osorio \& Palomo, 2004, citado por Villalobos, 2009, p.24). Y, por su parte, Ellis (2008) y Bouquet (2010) mencionan que el intento suicida es la conducta que una persona despliega con el fin de morir a pesar de los factores imprevistos que impidan el desenlace.

No obstante, el suicidio no es la única problemática a la que se enfrentan los jóvenes. Según la Organización Mundial de la Salud (OMS, 2009), también se enfrentan a los trastornos del comportamiento, la ansiedad, la depresión, el consumo de sustancias psicoactivas, las actitudes violentas, el alcoholismo y los trastornos ligados a la alimentación. Y, cabe resaltar que estos últimos, los trastornos alimenticios, son de gran interés debido a que constituyen un fenómeno social en aumento gracias a la combinación de factores de riesgo psicosociales, biológicos, culturales, genéticos, ambientales y familiares (Alba, 2010).

Actualmente, la idea del cuerpo perfecto ha alcanzado su máximo nivel de influencia gracias al respaldo de los medios 
de comunicación - Internet, televisión y revistas - y los nuevos criterios estéticos impuestos por la sociedad; $y$, en consecuencia, ha influenciado en la aparición de desajustes en relación con los hábitos de alimentación de los jóvenes (Galarsi, Correche \& Ledezma, 2010).

Según el Manual Diagnóstico y estadístico de los Trastornos Mentales -DSM 5-(APA, 2014), los trastornos de la conducta alimentaria y de la ingesta de alimentos son una alteración persistente en las conductas relacionadas con la alimentación; conllevan a una afectación en el consumo y absorción de los alimentos; y, a su vez, provocan un deterioro significativo en la salud física y en las diferentes áreas de funcionamiento de la persona afectada. Adicionalmente, la gravedad de las consecuencias de estos trastornos suele presentarse tanto en el área física como psicológica de la persona, pues suele manifestarse en enfermedades cardiacas, alteración de los mecanismos normales que ayudan a controlar el peso corporal, osteoporosis o pérdida de masa ósea, malnutrición debida a la carencia de nutrientes, muertes prematuras, elevada comorbilidad con el aislamiento social, baja autoestima, intenso miedo al rechazo o al abandono y, en los casos más graves, el suicidio (Portela de Santana, Da Costa Ribeiro Junior, Mora Giral \& Raich, 2012).

La anorexia y la bulimia se han expandido tan rápidamente en el mundo que pueden considerarse una verdadera epidemia social (Peláez, Raich \& Labrador, 2010 y se ha encontrado que los factores de riesgo para las perturbaciones en la conducta alimentaria varían según la tipología del trastorno - bulimia o anorexia-, sin embargo, la insatisfacción corporal y la depresión resultan ser comunes para ambos. Específicamente, para la anorexia nerviosa se han identificado algunos factores de riesgo como las críticas a la figura corporal, el perfeccionismo, las dietas especiales, ser mujer adolescente, tener baja autoestima y presentar una percepción distorsionada de la imagen corporal; mientras que para la bulimia nerviosa, además de los ya mencionados, se encuentra como factor de riesgo importante el temor a engordar (Rutsztein, Scappatura \& Murawski, 2014).

Ahora bien, la detección y atención del suicidio y de los trastornos alimentarios es una tarea complicada debido a la multitud de factores intervinientes y a la dificultad general que implica su abordaje; sobre todo, porque el manejo de los mismos requiere la conformación de un equipo de profesionales de diversas disciplinas que trabajen mancomunadamente (López \& Treasure, 2011).

Por otra parte, la asociación entre los trastornos de la alimentación y los fenómenos suicidas se ha investigado, según Vargas y Saavedra (2012), especialmente en estudios de revisión sistemática, donde se encuentra una asociación significativa entre intentos suicidas efectuados el último año y los trastornos alimentarios en adolescentes mujeres norteamericanas.

Siguiendo esta línea, para algunos autores el suicidio constituye la mayor causa de muerte en adolescentes con trastornos de alimentación, pues el porcentaje de muerte autoinflingida va desde el 1.8 hasta el $7.3 \%$ (Bodell, Joiner \& Keel, 2013; Cruzat, Ramírez, Melipillán \& Marzolo, 2008). Mientras que para otros, como Rodríguez, Rodríguez, Gempeler y Garzón (2013), aunque en personas con anorexia nerviosa el suicidio es la primera causa de muerte, cuando se presenta la bulimia, la ocurrencia de intentos suicidas es lo más común; y para otros, como Sánchez, Villareal-González, Musitu y Martínez (2010) y Forrest et al., (2016), existe una relación significativa entre los problemas alimentarios, la ideación suicida y otros trastornos emocionales, como la depresión.

En un estudio llevado a cabo con 2462 pacientes (95\% mujeres) admitidos por trastornos de conducta alimentaria - TCA - entre los años 1995-2010 en Estados Unidos, el $6.3 \%$ fueron internados por intento suicida, y se encontró que los riesgos de cometer suicidio aumentaban 4.7 veces ante la presencia del TCA $(O R, 4.7 ; 95 \% I C, 1.41-15.74)$, ocho veces ante la presencia de anorexia nerviosa $(O R, 8.01$; $95 \%$ CI, 5.40-11.87), y 5.08 veces en bulimia nerviosa (OR, 5.08; $95 \%$ CI, 3.46-7.42) (Suokas et al., 2014).

Por su parte, Suokas et al. (2013) encontraron resultados similares en un estudio previo para anorexia nerviosa $(O R$, $5.07 ; 95 \% C I, 1.87-18.84)$ y bulimia nerviosa $(O R, 6.07$; $95 \%$ CI, 2.47-14.89). Y Bodell et al. (2013), mediante un análisis de regresión, encontraron que la bulimia nerviosa predice significativamente la aparición e incremento del riesgo suicida y trastornos comórbidos; debido a que la anorexia nerviosa no fue significativa, sugieren desarrollar nuevos estudios que permitan dilucidar el papel causal de la bulimia nerviosa y su aplicabilidad en la evaluación del riesgo suicida (Toro, Vianchá, Martínez \& Pérez, en prensa).

Con respecto al estudio de la ideación suicida en adolescentes, Sánchez-Sosa et al. (2010) realizaron un estudio en el que se identificó una relación directa y significativa entre cuatro variables de estudio y la ideación suicida, de la siguiente manera: a) sintomatología depresiva $(\mathrm{r}=.511$; $\mathrm{p}=.001) ; \mathrm{b})$ conducta alimentaria de riesgo $(\mathrm{r}=.271 ; \mathrm{p}=$ $.001)$; c) victimización $(\mathrm{r}=.262 ; \mathrm{p}=.001) ; \mathrm{y}$ d) problemas de ajuste escolar $(r=.218 ; p=.001)$. Estos resultados apoyan la premisa de la ideación suicida como un fenómeno multifactorial y sugieren que los futuros trabajos científicos se encaminen en esta dirección.

En Colombia, estudios como los de Piñeros, Molano y López de Mesa (2010) dan cuenta de la comorbilidad entre los trastornos alimentarios con trastornos depresivos 
y ansiosos, así como con conductas suicidas, que son más frecuentes en adolescentes con diagnóstico clínico de TCA que en aquellos sin diagnóstico psiquiátrico o con otros trastornos $(\mathrm{p}=.019)$. Estos resultados muestran la importancia de evaluar el riesgo suicida y su relación con los TCA en jóvenes.

Sumado a lo anterior, en el departamento de Boyacá el grupo de edad que presenta mayor número de intentos de suicidio es el de los 15 años, edad también crítica para el desarrollo de trastornos alimentarios (Carmona et al., 2010). Y teniendo en cuenta el aumento en el número de suicidios y de trastornos de la conducta alimentaria que hay en la actualidad, estos dos son considerados problemas graves de salud pública (Fandiño, Giraldo, Martínez, Aux \& Espinosa, 2007; Manrique, Barrera \& Ospina, 2006).

Dichos problemas justifican la realización del presente estudio, cuyo objetivo general es el de establecer si existe alguna asociación entre los trastornos alimentarios y la conducta suicida en escolares de Boyacá, Colombia.

\section{MÉTODO}

\section{Tipo de estudio}

Se realizó un estudio selectivo probabilístico transversal que se enmarca en una estrategia descriptiva (Ato, López \& Benavente, 2013). El tipo de estudio es correlacional puesto que su finalidad fue identificar la relación existente entre los síntomas de los trastornos alimentarios y la conducta suicida en jóvenes (Hernández, Fernández \& Baptista, 2010). Los datos empíricos originales son elaborados por las autoras y se enmarcan dentro de la lógica epistemológica de tradición objetivista (Montero \& León, 2007).

\section{Participantes}

Los participantes fueron seleccionados mediante un muestreo probabilístico estratificado (Hernández et al., 2010), cuyas variables de estratificación fueron la ciudad, la institución educativa y el grado escolar. Los valores con los que se trabajó fueron $\mathrm{N}=85444, \mathrm{p}=.13, \mathrm{q}=.87, \mathrm{Z}=2.576$, $\mathrm{ye}=.05$. El tamaño muestral arrojado fue de 1500 estudiantes, pero tras la selección de los cuestionarios diligenciados adecuadamente, la muestra quedó conformada por 1292 estudiantes de octavo a undécimo grado de instituciones de educación media en las ciudades de Tunja, Duitama y Sogamoso del departamento de Boyacá, Colombia.

Como criterios de inclusión, se tuvieron en cuenta la voluntariedad y la firma del consentimiento y asentimiento informado. Finalmente, se contó con una muestra homogénea de 710 mujeres (55.03\%) y 582 hombres (44.96\%), cuyo rango de edad se ubicó entre los 12 y los 20 años, con un promedio de 15.4 años $(D T=1.35)$.

\section{Instrumentos}

\section{Cuestionario sociodemográfico.}

Las investigadoras del presente estudio elaboraron un cuestionario sociodemográfico que consistió en seis preguntas de carácter nominal y ordinal, el cual permitió indagar acerca de las variables sociodemográficas - tales como sexo, edad, grado, tipo de familia, ciudad y nombre del colegio- de cada uno de los participantes.

\section{Cuestionario Sick Control On Fat Food (SCOFF).}

El cuestionario SCOFF consta de cinco preguntas que abordan el núcleo fundamental de la bulimia y la anorexia. Su nombre deriva del acrónimo de las palabras en inglés de cada una de las preguntas (Morgan et al., 1999), y cuenta con una sensibilidad del $100 \%$ (IC $95 \% 96.9 \%$-100\%) una especificidad del $87 \%$ (IC $95 \%$, $79.2 \%$ - 93.4\%) en la población inglesa. Este instrumento fue validado en la ciudad en Bucaramanga, Colombia, por Rueda (2005), y las puntuaciones de 0-1 indican ausencia de trastorno alimenticio, de 2-3 implican riesgo, y de 4-5 señalan la presencia del mismo.

Inventario de ideación suicida positiva y negativa (PANSI).

Este instrumento fue adaptado por medio de la técnica de traducción simple. El PANSI (Positive and Negative Suicidal Ideation) de Osman et al. (1998) es un cuestionario de 14 ítems, de los cuales seis abordan la ideación suicida positiva (factores protectores), mientras que ocho de estos indagan sobre la ideación suicida negativa (factores de riesgo). Dichos factores son evaluados en el marco de las últimas dos semanas y en los ítems se pregunta qué tan a menudo la persona ha presentado cada uno de los 14 pensamientos.

Para responder al cuestionario, la persona hace uso de una escala de cuatro puntos que oscilan entre 0 (nunca) y 4 (siempre). Los ítems se derivan en dos escalas factoriales: ideación suicida positiva, donde el punto de corte es de 18 y los valores inferiores indican la presencia de riesgo, mientras que los valores superiores indican su ausencia; y la ideación suicida negativa, cuyo punto de corte es de 16 , donde los valores inferiores muestran riesgo suicida y los superiores ausencia de riesgo.

Este cuestionario ha mostrado coeficientes de consistencia interna superiores a .8 y una adecuada evidencia de validez de constructo (concurrente y discriminante) tanto en muestras clínicas como no-clínicas (Osman et al., 2003). 


\section{Procedimiento}

Inicialmente, se contactó a los secretarios de educación, rectores y coordinadores de los colegios públicos de las tres ciudades para solicitar su autorización; en cada una de las instituciones se indicó el número de participantes por grado $(8,9,10$ y 11$)$ de acuerdo al muestreo realizado, y se procedió a seleccionar al azar los estudiantes participantes. Posteriormente, se enviaron los consentimientos y asentimientos informados a los padres de familia para ser diligenciados y entregados el día de la aplicación del instrumento.

El día de la aplicación se le explicó a los estudiantes el objetivo del estudio y la forma en que se usaría la información suministrada, y se indicó el adecuado diligenciamiento del instrumento, el cual iniciaba con los datos sociodemográficos, seguido por el PANSI y posteriormente con el SCOFF. La aplicación se realizó de manera grupal y el diligenciamiento del inventario fue individual; en promedio, dicha aplicación tuvo una duración de 60 minutos. Finalmente, el análisis de resultados se realizó con el Software SPSS versión 20.

\section{Análisis de datos}

Una vez diligenciados los instrumentos, se elaboró una base de datos y se procedió al análisis de resultados obtenidos, específicamente mediante estadísticos descriptivos como frecuencias, medias, medianas, modas y desviaciones estándar. Posteriormente, de acuerdo con el carácter nominal de las variables, se realizaron las asociaciones entre las mismas.

\section{Consideraciones éticas}

Se solicitó la autorización a las directivas de los colegios públicos y se hizo entrega de consentimientos informados a padres de familia y asentimientos informados para los participantes; además, todo el procedimiento investigativo se ajustó a la legislación colombiana vigente de la resolución número 8430 de 1993 del Ministerio de Salud Nacional y de la ley 1090 de 2006 (Corte Constitucional, 2006), en las cuales se dictan las normas para la investigación en salud y la práctica de investigaciones en psicología. Finalmente, se contó con la aprobación del Comité de Bioética institucional. Se clarifica que en el presente estudio no existió ningún conflicto de intereses.

\section{RESULTADOS}

A continuación se muestran los datos de la presencia de ideación, acto suicida y trastornos alimentarios en la muestra participante, seguidos de las asociaciones halladas entre estas variables.

\section{Ideación suicida}

Los resultados encontrados revelan que de los 1292 jóvenes, el 43 \% (556) mostró presencia de ideación suicida positiva, es decir, bajas puntuaciones en factores protectores (véase Tabla 1).

Tabla 1

Presencia de ideación suicida positiva en la muestra total

\begin{tabular}{ccc}
\hline & Frecuencia & Porcentaje \\
\hline Sin riesgo & 736 & $57 \%$ \\
Con riesgo & 556 & $43 \%$ \\
Total & 1292 & $100 \%$ \\
\hline
\end{tabular}

Los resultados presentados en la Tabla 2 muestran que del total de jóvenes que participaron en el estudio, 230 $(17.8 \%)$ habían pensado en el suicidio como alternativa, mientras que $136(10.5 \%)$ no contaba con factores protectores que les permitieran hacer frente a las situaciones adversas (véase Tabla 2).

Tabla 2

Asociación entre ideación positiva e ideación negativa de la muestra total

\begin{tabular}{|c|c|c|c|c|}
\hline & \multicolumn{4}{|c|}{ Ideación positiva } \\
\hline & & $\begin{array}{c}\text { Sin } \\
\text { riesgo }\end{array}$ & $\begin{array}{l}\text { Con } \\
\text { riesgo }\end{array}$ & Total \\
\hline \multirow{4}{*}{$\begin{array}{l}\text { Ideación } \\
\text { negativa } \text { Con riesgo }\end{array}$} & Frecuencia & 642 & 420 & 1062 \\
\hline & $\%$ del total & $49.7 \%$ & $32.5 \%$ & $82.2 \%$ \\
\hline & Frecuencia & 94 & 136 & 230 \\
\hline & $\%$ del total & $7.3 \%$ & $10.5 \%$ & $17.8 \%$ \\
\hline \multirow{2}{*}{ Total } & Frecuencia & 736 & 556 & 1292 \\
\hline & $\%$ del total & $57.0 \%$ & $43.0 \%$ & $100.0 \%$ \\
\hline
\end{tabular}

\section{Intento suicida}

De los 1287 jóvenes que respondieron a la pregunta "Alguna vez ha intentado suicidarse", el $15.38 \%$ (198 estudiantes) manifestó que sí lo había hecho, dato que resulta relevante dada su alta frecuencia de manifestación (véase Tabla 3).

Tabla 3

Intentos suicidas en los estudiantes por sexo

\begin{tabular}{ccc}
\hline Intento de suicidio & Frecuencia & $\begin{array}{c}\text { Porcentaje } \\
\text { dentro de intento }\end{array}$ \\
\hline Sí ha intentado suicidarse & 198 & 15.38 \\
Mujeres & 119 & 60.1 \\
Hombres & 79 & 39.1 \\
\hline
\end{tabular}

\section{Síntomas de trastornos alimentarios}

Se consideró como "riesgo" a las puntuaciones entre 2 y 3 , que indican la aparición de algunas conductas relacionadas 
con trastornos alimentarios; y se denominó "presencia" a las puntuaciones entre 4 y 5 , que indican la manifestación de conductas características de los trastornos alimentarios.

Con esto, el $2.6 \%$ (33 estudiantes) del total de los participantes mostró indicadores para un posible trastorno alimentario - anorexia o bulimia-; y el 21.6\% (279 estudiantes) podía estar en riesgo de alguno de estos trastornos, siendo las mujeres quienes puntuaron frecuencias más altas.

Tabla 4

Características sociodemográficas de los estudiantes con sintomatología asociada a los trastornos alimentarios

\begin{tabular}{cccc}
\hline & \% Ausencia & \% Riesgo & \% Presencia \\
\hline Ciudad & & & \\
Tunja & 77.6 & 20.6 & 1.8 \\
Duitama & 71.5 & 25.0 & 3.5 \\
Sogamoso & 77.0 & 20.4 & 2.6 \\
Sexo & & & \\
Femenino & 70.2 & 26.4 & 3.4 \\
Masculino & 82.3 & 16.3 & 1.4 \\
Edad & & & \\
12 & 100 & 0.0 & 0.0 \\
13 & 73.8 & 21.5 & 4.6 \\
14 & 78.7 & 18.9 & 2.4 \\
15 & 76.7 & 20.2 & 3.1 \\
16 & 73.1 & 24.7 & 2.2 \\
17 & 71.5 & 24.8 & 3.6 \\
18 & 81.5 & 18.5 & 0.0 \\
19 & 78.9 & 21.1 & 0.0 \\
20 & 100.0 & 0.0 & 0.0 \\
Grado & & & 2.8 \\
8 & 79.0 & 18.0 & 3.0 \\
9 & 76.8 & 20.3 & 2.9 \\
10 & 73.5 & 23.7 & \\
11 & 72.4 & 26.0 & \\
\hline
\end{tabular}

En resumen, se encontró un mayor riesgo y presencia de trastornos alimentarios en la ciudad de Duitama (28.5\%), en población femenina (28.5\%), de 17 años $(28.4 \%)$, y de grado once $(27.6 \%)$ (véase Tabla 4$)$.

\section{Ideación suicida y trastornos alimentarios}

Para identificar los trastornos alimentarios se aplicó el cuestionario SCOFF, el cual permite realizar un tamizaje del riesgo frente al desarrollo de un trastorno alimentario, pero no identifica el tipo de trastorno específico exhibido por la persona. Los resultados presentados en la Tabla 5 establecen que de los 33 estudiantes que evidenciaron un trastorno alimentario, 14 de ellos (42.4\%) también presentaban ideación suicida.
Tabla 5

Asociación entre trastornos alimentarios e ideación suicida

\begin{tabular}{ccccc}
\hline \multirow{2}{*}{ SCOFF } & \multicolumn{2}{c}{ PANSI } & Total \\
\hline \multirow{2}{*}{ Ausencia } & $\begin{array}{c}\text { Frecuencia } \\
\% \text { dentro de } \\
\text { SCOFF }\end{array}$ & 835 & 131 & 966 \\
& $\begin{array}{c}\text { Frecuencia } \\
\text { Riesgo }\end{array}$ & 198 & $13.6 \%$ & $100.0 \%$ \\
& $\begin{array}{c}\% \text { dentro de } \\
\text { SCOFF }\end{array}$ & $71.0 \%$ & $29.0 \%$ & $100.0 \%$ \\
Presencia & $\begin{array}{c}\text { Frecuencia } \\
\% \text { dentro de } \\
\text { SCOFF }\end{array}$ & 19 & 14 & 33 \\
Total & $\begin{array}{c}\text { Frecuencia } \\
\% \text { dentro de } \\
\text { SCOFF }\end{array}$ & 1052 & $42.4 \%$ & $100.0 \%$ \\
\hline
\end{tabular}

El puntaje $c h i^{2}$ fue de 49.8 y el sig bilateral, de .00; resultados que indican una asociación significativa entre los trastornos alimentarios y la ideación suicida. Como se observa en la Tabla 5, el $42.4 \%$ de quienes presentaron sintomatología relacionada con trastornos alimentarios también afirmaron presentar ideación suicida negativa (riesgo); además, 81 estudiantes - que constituyen el $29.0 \%$ de los jóvenes en riesgo de presentar un trastorno alimentario - presentó ideas negativas.

\section{Intento suicida y trastornos alimentarios}

Como se observa en la Tabla 6, el $6.0 \%$ de quienes manifestaron presencia de sintomatología relacionada con trastornos alimentarios reportó haber intentado suicidarse. Así mismo, el $30.5 \%$ de los jóvenes en riesgo de presentar un trastorno alimentario afirmó haber intentado suicidarse.

Tabla 6.

Contingencia entre trastornos alimentarios e intención suicida

\begin{tabular}{|c|c|c|c|c|}
\hline \multicolumn{2}{|c|}{ Trastornos alimentarios } & $\begin{array}{l}\text { Ha intentado } \\
\text { suicidarse }\end{array}$ & $\begin{array}{c}\text { No ha } \\
\text { intentado } \\
\text { suicidarse }\end{array}$ & Total \\
\hline \multirow[b]{2}{*}{ Ausencia } & \multirow{2}{*}{$\begin{array}{l}\text { Frecuencia } \\
\% \text { dentro de } \\
\text { Acto }\end{array}$} & 127 & 836 & 963 \\
\hline & & $63.5 \%$ & $77.8 \%$ & $75.5 \%$ \\
\hline \multirow[b]{2}{*}{ Riesgo } & \multirow{2}{*}{$\begin{array}{l}\text { Frecuencia } \\
\% \text { dentro de } \\
\text { Acto }\end{array}$} & 61 & 218 & 279 \\
\hline & & $30.5 \%$ & $20.3 \%$ & $21.9 \%$ \\
\hline \multirow[b]{2}{*}{ Presencia } & \multirow{2}{*}{$\begin{array}{l}\text { Frecuencia } \\
\% \text { dentro de } \\
\text { Acto }\end{array}$} & 12 & 21 & 33 \\
\hline & & $6.0 \%$ & $2.0 \%$ & $2.6 \%$ \\
\hline \multirow[b]{2}{*}{ Total } & \multirow{2}{*}{$\begin{array}{c}\text { Frecuencia } \\
\% \text { dentro de } \\
\text { Acto }\end{array}$} & 200 & 1075 & 1275 \\
\hline & & $100.0 \%$ & $100.0 \%$ & $100.0 \%$ \\
\hline
\end{tabular}


El puntaje $c h i^{2}$ fue de 23.263 y el sig bilateral, de .00; resultados que indican asociación significativa entre los trastornos alimentarios y la intención suicida.

Por otro lado, a través del coeficiente $r$ de Spearman se estableció la correlación entre el total del SCOFF —que evalúa los trastornos alimentarios-y los totales de las dos escalas del PANSI — positivo y negativo-, y se encontró una correlación baja, pero significativa, entre los trastornos alimentarios y el PANSI negativo: el puntaje del rs fue $=.19$, con un valor $p=.00$, lo que indica que a mayor presencia de señales de alarma de trastornos alimentarios, más factores de riesgo de ideación suicida.

\section{DISCUSIÓN}

Los resultados encontrados indican una relación entre los trastornos alimentarios y la ideación e intento suicida; específicamente, 230 participantes -que corresponden al $17.8 \%$ del total de la muestra - presentaron riesgo de ideación suicida negativa (factores de riesgo), aspecto que se relaciona con el fracaso, la desesperanza, la baja autoeficacia, la baja autoestima, la frustración, la tristeza, la dificultad en la resolución de problemas y las estrategias de afrontamiento poco adaptativas focalizadas en la emoción, las cuales tienden a la autoculpabilización y se relacionan con experiencias de vida que pueden ser experimentadas por los jóvenes en la cotidianidad (Kirchner, Ferrer, Forns \& Zanini, 2011; Miranda, Cubillas, Román \& Abril, 2009; Montes \& Montes 2009; Quintanilla, Valadez, Valencia \& González, 2005; Villalobos, 2009).

Según los resultados del presente estudio, cabe resaltar que de los 1292 jóvenes, el $43 \%$ —correspondiente a 556 participantes - resultó ser vulnerable al suicidio, hallazgo que corrobora lo encontrado en otras investigaciones que indican que la ideación suicida es un factor predictor o de riesgo de suicidio (Cabra, Infante \& Sossa, 2010; Cano, Gutiérrez \& Nizama, 2009; Córdova, Estrada, \& Velázquez, 2013).

Según Estruch y Cardús (1982), debido a que el suicidio es una problemática multicausal, las razones o causas por las cuales un individuo intenta suicidarse pueden ser tan variadas y diferentes que explicarlas en su totalidad resultaría demasiado arduo; no obstante, mencionan que existe una vinculación entre el suicidio y los trastornos de la alimentación, aspecto que es confirmado por los resultados de esta investigación.

En cuanto a la variable sexo, según el Forensis 2015 (INML, 2015), en Colombia son las mujeres las que, en mayor proporción, intentan suicidarse, pero son los hombres quienes obtienen los resultados más letales. Según este informe, para el año 2015 se suicidaron 1655 varones -es decir, el $80.03 \%$ del total de suicidios-, mientras que el número de casos en mujeres fue mucho menor -413 suicidios consumados- Estos datos concuerdan con lo encontrado en esta investigación, dado que el $60.1 \%$ de las personas que habían intentado suicidarse correspondió al sexo femenino, mientras que el $39.9 \%$ restante era de sexo masculino.

La información recolectada con el cuestionario SCOFF indica que el $2.6 \%$ - equivalente a 33 adolescentes - exhibe indicadores para un posible trastorno alimentario; mientras que el $21.9 \%$ —correspondiente a 279 jóvenes - puede estar en riesgo de presentar bulimia o anorexia, siendo las mujeres quienes registraron frecuencias más altas. Con respecto a esto, según los estudios de Kiefer, Leitner, Bauer y Rieder (2000), las mujeres tienden a orientar la percepción de sí mismas hacia el patrón de belleza social y están más influenciadas que los hombres por el ideal de delgadez; por lo tanto, más a menudo están insatisfechas con su peso y se perciben considerablemente más pesadas de lo que realmente están. Lo anterior, sumado a la excesiva atención que se da a la imagen corporal y al peso, resultan ser factores de riesgo para el desarrollo de un trastorno alimentario.

El presente estudio corrobora que el sexo femenino presenta mayores porcentajes de síntomas de trastornos alimentarios, pues el $26.4 \%$ de las participantes se encontró en riesgo, y el $3.4 \%$ presentó dicha problemática. En el sexo masculino, estos porcentajes correspondieron al $16.3 \%$ y al $1.4 \%$, respectivamente; resultados que ratifican las aseveraciones realizadas por autores como Sherman y Thompson (2005), quienes identificaron a la mujer como la principal víctima de los trastornos de alimentación; esto teniendo en cuenta que la sociedad, sobre todo en los medios visuales e impresos, contribuye a la preocupación por la esbeltez y a percibir el éxito y la felicidad como consecuencias de la figura (Cano et al., 2007; Nuño, Celis \& Unikel, 2009; Sánchez, Guzmán \& Cáceres, 2005).

Por otra parte, padecer algún trastorno alimentario y tener comportamientos suicidas ha sido asociado también con características o trastornos de personalidad (Borrego, 2010; Galarsi et al., 2010), aspecto que no fue evaluado en esta investigación, pero que debe considerarse en próximos estudios con el fin de obtener más información y establecer posibles relaciones entre dichas variables.

El intento suicida y la ideación suicida son indistintamente comunes en mujeres con trastornos alimentarios y con depresión (García-Valencia et al., 2008; Medina, 
Piernagorda \& Rengifo, 2010). En población clínica, por ejemplo, la autolesión y las diferentes formas de esta - como los trastornos alimentarios - correlacionan con el suicidio (Negredo, Melis \& Herrero, 2011). Los resultados de la presente investigación muestran que aunque la población objeto de estudio no era clínica, también hubo una asociación significativa entre el intento e ideación suicida con los trastornos alimentarios; indicador que resalta la necesidad de profundizar en este aspecto para prevenir la conducta suicida en los jóvenes.

En este sentido, es importante afirmar que el intento suicida y los trastornos alimentarios influyen directa 0 indirectamente en las conductas lesivas o autolesivas directas - como los cortes de piel, morderse gravemente las uñas o golpearse, entre otras- e indirectas - como el alcoholismo, conducir de manera peligrosa, el tabaquismo, la drogadicción y los trastornos alimentarios- (Zannety, Santonastaso, Sgarabatty, Degortes \& Favaro, 2013). Para Zanety et al. (2013) tanto los trastornos alimentarios como las conductas autolesivas comparten puntos importantes, como que son típicos en mujeres, que su comienzo se produce durante la adolescencia - lo cual se corrobora con los resultados del presente estudio- y que pueden estar vinculados con la insatisfacción corporal y el fuerte sentido de ineficacia que a menudo implica el autocastigo.

Desde esta perspectiva, los resultados obtenidos en cuanto a la relación significativa y positiva entre la ideación e intento suicida con los trastornos alimentarios apoyan los hallazgos de otros estudios, como los de Laufer, (1995); Machado, Goncalves, Machado, Torres y Brandao (2004); Rodríguez et al. (2007); Cano et al. (2007); Cortés, Díaz, Mejía y Mesa (2003); Breton, Gutiérrez, Villatoro, Moreno \& Gaytan (2009); Nuño et al. (2009), Sánchez-Sosa et al. (2010); Vargas y Saavedra (2012); y Forrest et al., (2016); lo que permite evidenciar la correspondencia existente entre estas dos condiciones psicológicas y la necesidad de continuar indagando al respecto.

Por su carácter transversal y el tipo de estadísticos utilizados, el presente estudio no da lugar a conclusiones que permitan predecir el comportamiento futuro de los jóvenes adolescentes. Por ello, se considera pertinente que, desde un enfoque diferencial (Caycedo et al., 2010) y con el concurso de padres de familia, entidades educativas e instituciones de apoyo social o de salud deberían elaborar e implementar estrategias de intervención orientadas a fortalecer en los escolares características como la autoestima, el sentido de autocontrol y la autoconfianza, así como la estabilidad del vínculo afectivo y del apoyo sociofamiliar; todo esto con el fin de favorecer los factores protectores frente a la conducta suicida y los trastornos alimentarios.

\section{REFERENCIAS}

Alba, L. (2010). Salud de la adolescencia en Colombia: bases para una medicina de prevención. Revista Universitas Médica, 51(1), 29-42. Recuperado de http://med.javeriana. edu.co/publi/vniversitas/serial/V51n1/Universitas\%20 M\%E9dica\%20Vol.\%2051\%20No.\%201/Salud\%20 de\%20la\%20adolesce.pdf.

American Psychiatric Association. (2014). Manual Diagnóstico y estadistico de los Trastornos Mentales, DSM-5. Washington, D.C.: Editorial Médica Panamericana.

Ato, M., López, J. J., \& Benavente, A. (2013). Un sistema de clasificación de los diseños de investigación en psicología. Anales de psicología, 29(3), 1038-1059. doi:10.6018/analesps.29.3.178511.

Ballesteros, M., Gutiérrez, M., Sánchez, L., Herrera, N., Gómez, A., \& Bouquet, R. (2010). El suicidio en la juventud: Una mirada desde la teoría de las representaciones sociales. Revista Colombiana de Psiquiatría, 39(3), 523543. Recuperado de http://www.redalyc.org/articulo. oa?id=80619187006.

Bodell L., Joiner T., \& Keel P. (2013) Comorbidity-independent risk for suicidality increases with bulimia nervosa but not with anorexia nervosa. Journal of Psychiatric, 47(5), 617-621.

Borrego, C. (2010). Factores e indicadores clínicos asociados a los trastornos de la conducta alimentaria. Revista de Psicología, 12(1), 13-50. Recuperado de http://sisbib.unmsm. edu.pe/BVRevistas/rev_psicologia_cv/v12_2010/pdf/ a02.pdf.

Bouquet, R. (2010). El suicidio en la juventud: Una mirada desde la teoría de las representaciones sociales. Revista Colombiana de Psiquiatría, 39(3), 523-543.

Breton, M., Gutiérrez, M., Villatoro, J., Moreno, M., \& Gaytan, L. (2009). Intento suicida y depresión como factores relacionados con posible trastorno de la conducta alimentaria. Recuperado de http://www.uade.inpsiquiatria.edu.mx/pagina_contenidos/cuadernillos/carteles_macro/Michelle. pdf.

Cabra, O., Infante, D., \& Sossa, F. (2010). El suicido y los factores de riesgo asociados en niños y adolescentes. Revista Médica Sanitas, 13(2), 28-35. Recuperado de http://www. unisanitas.edu.co/revista/18/artículos/suicidio.pdf.

Cano, A., Castaño, J., Corredor, D., García, A., Gonzales, M., Lloreda, O., Lucero, K., Ortiz, J. Páez, M., Patiño, L., Pérez, L., Villegas L \& Zuluaga, A. (2007). Factores de riesgo para trastornos de la alimentación en los alumnos de la Universidad de Manizales. Revista de Medicina UNAB, 10(3), 117129. Recuperado de http://revistas.unab.edu.co/index.ph p?journal $=$ medunab\&page $=$ article $\&$ op $=$ view $\&$ path $\% 5 B$ $\% 5 \mathrm{D}=97 \&$ path $\% 5 \mathrm{~B} \% 5 \mathrm{D}=88$.

Cano, P., Gutiérrez, C., \& Nizama, M. (2009). Tendencia a la violencia e ideación suicida en adolescentes escolares en una ciudad de la Amazonía peruana. Revista Peruana de 
Medicina Experimental y Salud Publica, 26(2), 175-181. Recuperado de http://www.scielo.org.pe/scielo.php?script=sci_arttext\&pid=S1726-463420090002 00007\&ln$\mathrm{g}=\mathrm{es} \& \mathrm{nrm}=\mathrm{iso}>$. ISSN 1726-4634.

Carmona, A., Arango, A., Castaño, J., Escobar, J., García, C., $\&$ Godoy, S. (2010). Caracterización del intento de suicidio en una población integrada a un hospital infantil de Manizales (Caldas-Colombia). 2000-2008. Archivos de Medicina, 10(1), 9-18. Recuperado de http://www.redalyc.org/ pdf/2738/273819493001.pdf.

Casullo, M., Bonaldi, D., \& Fernández, M. (2000). Comportamientos suicidas en la adolescencia: morir antes de la muerte. Buenos Aires: Editorial Lugar.

Caycedo, A., Arenas, M., Benítez, M., Cavanzo, P., Leal, G., \& Guzmán Y. (2010). Características psicosociales y familiares relacionadas con el intento suicida en una población adolescente en Bogotá 2009. Persona Bioética. Recuperado de http://personaybioetica.unisabana.edu.co/index.php/personaybioetica/article/view/1807/2424.

Córdova, A., Estrada, A., \& Velázquez, M. (2013). Detección de riesgo suicida en una muestra de adolescentes estudiantes de escuelas secundarias. Revista Intercontinental de Psicología y Educación, 15(1), 7-21.

Córdova, M., Cubillas, M., \& Román, R. (2011). ¿Es posible prevenir el suicidio? Evaluación de un programa de prevención en estudiantes de bachillerato. Pensamiento Psicológico, 9(17), 21-32. Recuperado de http:// www.scielo.org.co/scielo.php?script $=$ sci_arttext\&pi$\mathrm{d}=$ S1657-89612011000200003\&lng=en\&tlng=es.

Corte Constitucional. (2006). Ley 1090 de 2006. Recuperado de http://www.psicologiaprospectiva.com/introley1090. html.

Cortés, A. (2014). Conducta suicida adolescencia y riesgo. Revista Cubana de Medicina General Integral, 30(1), 132-139. Recuperado de http://scielo.sld.cu/scielo.php?script=sci_arttext\&pid $=$ S0864=21252014000100013-\&lng=es\&tlnges.

Cortés, H., Díaz, A., Mejía, C., \& Mesa, J. (2003). Trastornos de la alimentación: Su prevalencia y principales factores de riesgo - estudiantes universitarias de primer y segundo año. Revista CES MEDICINA, 17 (3), 97-125.

Cruzat, M., Ramírez, C., Melipillán, R., \& Marzolo, P. (2008). Trastornos alimentarios y funcionamiento familiar percibido en una muestra de estudiantes secundarias de la comuna de concepción. Psykhe, (1), 81-90.

Ellis, T. (2008). Cognición y suicidio. Teoría, Investigación Terapia. Ciudad de México: Editorial Manual Moderno.

Estruch, J., \& Cardús, S. (1982). Los Suicidios. Barcelona: Editorial Herder.

Fandiño, A., Giraldo, S., Martínez, C., Aux, C., \& Espinosa, R. (2007). Factores asociados con los trastornos de la conducta alimentaria en estudiantes universitarios en Cali, Colombia. Colombia Médica, 38(4), 344-351. Recuperado de http://www.redalyc.com/src/inicio/ArtPdfRed.jsp?iC$v e=28338402 \#$.

Forrest L, Bodell, L., Witte, T., Goodwin, N., Bartlett, M., Siegfried, N., Eddy, K., Thomas, J., Franko, D., \& Smith, A. (2016). Association between eating disorder symptoms and suicidal ideation through thawarted belongingnnes and perceived burdersomenness among eating disorder patients. Journal of Affective Disorders, 195 (1), 127-35. Recuperado de https://www.ncbi.nlm.nih.gov/pubmed/26895090.

Gaete, V. (2015). Desarrollo psicosocial del adolescente. Revista Chilena de Pediatría, 86(6), 436-443. Recuperado de http://ac.els-cdn.com/S0370410615001412/1-s2.0S0370410615001412-main.pdf? tid=adc4cb46-084e11e7-a7d6-0000aacb361\&acdnat $=1489452147 \_33 d 973$ 9614753dade70c40391f0d37cf.

Galarsi, M., Correche, F., \& Ledezma, S. (2010). Conductas y actitudes hacia la alimentación en una muestra de mujeres jóvenes. Fundamentos en Humanidades, 11(21), 193-205. Recuperado de http://redalyc.uaemex.mx/redalyc/src/inicio/ArtPdfRed.jsp?iCve=18415426013\#.

Galdó, G. (2008). Atención al Adolescente. Cantabria: Editorial de la Universidad de Cantabria.

García-Valencia, J., Palacio-Acosta, C., Diago, J., Zapata, C., López, G. Ortiz, J., \& López, M. (2008). Eventos vitales adversos y suicidio: un estudio de autopsia psicológica en Medellín, Colombia. Revista Colombiana de Psiquiatría, 37(1), 11-28. Recuperado de http://www.scielo.org.co/ $\mathrm{pdf} / \mathrm{rcp} / \mathrm{v} 37 \mathrm{n} 1 / \mathrm{v} 37 \mathrm{n} 1 \mathrm{a} 02 . p d f$.

Hernández, R., Fernández, C., \& Baptista, P. (2010). Metodología de la Investigación. México: Editorial Mc Graw Hill.

Instituto Nacional de Medicina Legal y Ciencias Forenses (INML). (2012). Forensis. Recuperado de http://www. medicinalegal.gov.co/documents/10180/34861/6+5+su icidio+forensis+2012.pdf/654be518-60d0-4979-b899f26c83d1bb8c.

Instituto Nacional de Medicina Legal y Ciencias Forenses (INML). (2015). Forensis 2015. Recuperado de http://www. medicinalegal.gov.co/documents/88730/3418907/7.+SU ICIDIOS.pdf/01c41af2-27cf-4932-ae14-234d1eeaf425.

Kiefer, I., Leitner B., Bauer, R., \& Rieder, A. (2000). Body weight: The male and female perception. Preventive-medicine, 45(6), 274-8.

Kirchner, T., Ferrer, L., Forns, M., \& Zanini, D. (2011). Conducta autolesiva e ideación suicida en estudiantes de Enseñanza Secundaria Obligatoria. Diferencias de género y relación con estrategias de afrontamiento. Actas España Psiquiatría, 39(4), 226-35. Recuperado de http://actaspsiquiatria.es/ repositorio/13/72/ESP/13-72-ESP-226-235-920269.pdf.

Laufer, M. (1995). El adolescente suicida. Madrid: Editorial Biblioteca Nueva.

López, C., \& Teasure, J. (2011) Trastornos de la Conducta Alimentaria en adolescentes: Descripción y manejo. Revista Médica de Clínica Las Condes, 22(1), 85-97. Recu- 
perado de http://www.clinicalascondes.cl/Dev_CLC/ media/Imagenes/PDF \%20revista \%20m \%C3 \%A9dica/2011/1 \%20enero/10_PS_Lopez-12.pdf.

Machado, P., Goncalves, S., Machado, B., Torres, R., \& Brandao, I. (2004). Intentos de suicidio y la severidad en los trastornos alimentarios. Terapia Psicológica, 22(1), 57-60. Recuperado de http://repositorium.sdum.uminho.pt/bitstream/1822/3021/1/TerapiaPsicologica2004.pdf.

Manrique, F., Barrera, L., \& Ospina, J. (2006). Prevalencia de Bulimia y Anorexia en Adolescentes de Tunja y algunos Factores de Riesgo Probablemente Asociados. Revista Hacia la Promoción de la Salud, 18(2), 55-66. Recuperado de http://www.scielo.org.co/scielo.php?script=sci_arttext\&pid=S0121-5772013000200005.

Medina, O., Piernagorda, D., \& Rengifo, A. (2010). Prevalencia del suicidio en adultos jóvenes del departamento del Quindío-Colombia, durante el periodo 1989-2008. Pensamiento psicológico, 8(15), 9-16.

Ministerio de Salud Nacional. (1993). Resolución número 8430 de 1993. Recuperado de https://www.minsalud.gov.co/sites/rid/Lists/BibliotecaDigital/RIDE/DE/DIJ/RESOLUCION-8430-DE-1993.PDF.

Miranda, T., Cubillas, R., Román, P., \& Abril, V. (2009). Ideación suicida en población escolarizada infantil: factores psicológicos asociados. Revista Salud Mental, 32(6), 495-502.

Montero, I. \& León O. (2007). A guide for naming research studies in Psychology. Health Psychology, 7(3), 847-862.

Montes, G., \& Montes, F. (2009). El pensamiento social sobre el suicidio en estudiantes de bachillerato. Enseñanza e Investigación en Psicología, 14(2) 311-324. Recuperado de http://www.redalyc.org/pdf/292/29211992007.pdf.

Morgan, J., Reid, F., \& Lacey, J. (1999). The SCOFF questionnaire: Assessment of a new screening tool for eating disorder. BMJ Journals, 319(7223),1467-1468. Recuperado de https://www.ncbi.nlm.nih.gov/pubmed/10582927.

Negredo, L., Melis, F., \& Herrero, O. (2011). Factores de riesgo de la conducta suicida en internos con trastorno mental grave. Madrid: Ministerio del Interior. Secretaría General Técnica. Recuperado de http://www.interior.gob.es/documents/642317/1201664/Factores+de+riesgo+de+la+cond ucta + suicida + en + internos + con+trastorno+mental+grave +\%28NIPO+126-11-051-1\%29.pdf/f3efbc07-5caa-46018248-6147b79a6440.

Nuño, B., Celis, A., \& Unikel, C. (2009). Prevalencia y factores asociados a las conductas alimentarias de riesgo en adolescentes escolares de Guadalajara según sexo. Revista de Investigación Clínica, 61(4), 286-293. Recuperado de http:// www.artemisaenlinea.org.mx/acervo/pdf/revista_ADM/ Prevalencia \%20y \%20factores.pdf.

Organización Mundial de la Salud (OMS). (2009). El suicidio, un problema de salud pública enorme y sin embargo prevenible, según la OMS. Recuperado de http://www.who. int/mediacentre/news/releases/2004/pr61/es/index.html.
Osman, A., Gutiérrez, P., Kopper, B., Barrios, F. \& Chiros, C. (1998). The positive and negative suicide ideation inventory: Development and validation. Psychological Reports, 82, 783-793.

Osman, A., Gutiérrez, P., Jiandani J., Kopper B., Barrios, F., Linden S., \& Truelove, R. (2003). A preliminary validation of the Positive and Negative Suicide Ideation (PANSI) Inventory with normal adolescent samples. Journal of Clinical Psychology, 59(4), 493-512. Recuperado de http:// www.ncbi.nlm.nih.gov/pubmed/12652640.

Peláez M., Raich R., \& Labrador, F. (2010). Eating disorders in Spain: Revision of empirical epidemiological studies. Revista mexicana de trastornos alimentarios, 1(1), 62-75. Recuperado de http://www.scielo.org.mx/scielo.php?script=sci_arttext\&pid=S2007$15232010000100007 \& \operatorname{lng}=$ es\&tlng=en.

Piñeros, S., Molano J., \& López de Mesa, C. (2010). Factores de riesgo de los trastornos de la conducta alimentaria en jóvenes escolarizados en Cundinamarca (Colombia). Revista Colombiana de Psiquiatría, 39(2), 313-328. Recuperado de http://www.scielo.org.co/scielo.php?script=sci arttext\&pid=S0034-74502010000200007\&lng=en\&tlng= es.

Portela de Santana, M., Da Costa Ribeiro Junior, H., Mora Giral, M., \& Raich, R. M. ${ }^{a}$. (2012). La epidemiología y los factores de riesgo de los trastornos alimentarios en la adolescencia: una revisión. Nutrición Hospitalaria, 27(2), 391-401. Recuperado de http://scielo.isciii.es/scielo.php?script=sci ar ttext\&pid=S0212-16112012000200008\&lng=es\&tl$\mathrm{ng}=\mathrm{es}$.

Portilla, S. (2006). Bases teóricas de la psiquiatría infantil. Manizales, Colombia: Universidad de Caldas, Comité Editorial.

Quintanilla, R., Valadez, I., Valencia, J. F \& González. (2005). Tipos de personalidad y conducta suicida. Revista Investigación en salud, 6(2), 108-113. Recuperado de http://redalyc.uaemex.mx/pdf/142/14260209.pdf

Quintero, F., \& Piñeros, S. (2010). Estado del arte de las investigaciones sobre adolescentes y jóvenes en el departamento de Boyacá 1999-2009. Recuperado de https://es.scribd. com/document/208939824/ESTADO-DEL-ARTE-PRODUCCION-JUVENTUD-1999-2009-pdf.

Rodríguez G., Gempeler R., Pérez R., Solano S., Meluk, A., Guerrero, E., \& Liemann, E. (2007). Entre el sufrimiento interno y las palabras silenciadas: análisis de narrativas de pacientes con trastornos del comportamiento alimentario, trauma y automutilaciones. Revista Colombiana de Psiquiatría, 34, 237-254. Recuperado de http://redalyc.uaemex. $\mathrm{mx} /$ redalyc/src/inicio/ArtPdfRed.jsp?iCve=80636207\#.

Rodríguez, M., Rodríguez, N., Gempeler, J., \& Garzón, D. (2013) Factores asociados con intento de suicidio y comportamientos de autolesión no suicida en pacientes con trastornos del comportamiento alimentario. Revis- 
ta Colombiana de psiquiatría, 13, 19-26. doi: 10.1016/j. rcp.2013.11.007.

Rueda, G. (2005). Validación del cuestionario SCOFF para los trastornos de alimentación en Colombia. Avances en Psiquiatría Biológica, 7, 20-36.

Rutsztein, G., Scappatura, L., \& Murawski, B. (2014). Perfeccionismo y baja autoestima a través del continuo de los trastornos alimentarios en adolescentes mujeres de Buenos Aires. Revista Mexicana de Trastornos Alimentarios, 5(1), 39-49.

Sánchez, R., Guzmán. O., \& Cáceres, A. (2005). Estudio de la imitación como factor de riesgo para ideación suicida en estudiantes universitarios adolescentes. Revista Colombiana de Psiquiatría, 34(1), 12-25.

Sánchez-Sosa, J., Villarreal-González, M., Musitu, G., \& Martínez, B. (2010). Ideación Suicida en Adolescentes: Un Análisis Psicosocial. Psychosocial Intervention, 19(3), 279287. Recuperado de http://scielo.isciii.es/scielo.php?scrip$\mathrm{t}=$ sci_arttext\&pid=S1132-05592010000300008\&1ng $=$ es\&tlng=es.

Sherman \& Thompson (2005) Bulimia: Una guía para familiares y amigos. México: Editorial Trillas.

Suokas, J. T., Suvisaari, J. M., Gissler, M., Löfman, R., Linna, M. S., Raevuori, A., \& Haukka, J. (2013). Mortality in eating disorders: A follow-up study of adult eating disorder patients treated in tertiary care, 1995-2010. Psychiatry Research, 210(3), 1101-1106. doi:10.1016/j.psychres.2013.07.042.
Suokas, J. T., Suvisaari, J. M., Grainger, M., Raevuori, A., Gissler, M., \& Haukka, J. (2014). Suicide attempts and mortality in eating disorders: a follow-up study of eating disorder patients. General Hospital Psychiatry, 36(3), 355357. doi:10.1016/j.genhosppsych.2014.01.002.

Toro, R., Vianchá, M., Martínez, L., \& Pérez, M. (en prensa). Conducta suicida en Boyacá: factores psicosociales y prevención. Tunja: Ediciones Universidad de Boyacá.

Vargas, H., \& Saavedra, J. (2012). Factores Asociados con la conducta suicida en adolescents. Revista de Nueropsquiatría, 75(1), 19-28. Recuperado de http://www.upch.edu. $\mathrm{pe} / \mathrm{vrinve} /$ dugic/revistas/index.php/RNP/article/viewFile/1539/1567.

World Health Organization (WHO). (2012). For which strategies of suicide prevention is there evidence of effectiveness? Copenhagen, Denmark: WHO Regional Office for Europe.

Villalobos, F. (2009). Ideación suicida en jóvenes: formulación $y$ validación del modelo integrador explicativo en estudiantes de educación secundaria y superior (Tesis doctoral). Universidad de Granada, España. Recuperado de http:// hera.ugr.es/tesisugr/1805982x.pdf.

Zannety, T., Santonastaso, P., Sgarabatty, E., Degortes, D., \& Favaro, A. (2013). Clinical and temperamental correlates of body image disturbance in eating disorders. The Journal of Eating Disorders Association, 21(1), 32-47. Recuperado de http://cirrie.buffalo.edu/database/204823. 